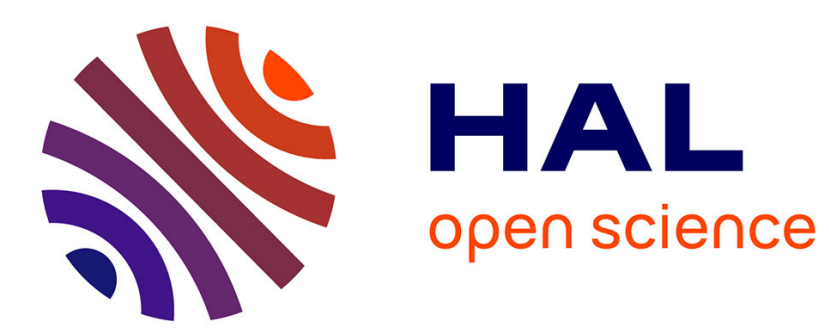

\title{
An extensive study on LES, RANS and hybrid RANS/LES simulation of a narrow-gap open Taylor-Couette flow
}

Christophe Friess, Sébastien Poncet, Stéphane Viazzo

\section{To cite this version:}

Christophe Friess, Sébastien Poncet, Stéphane Viazzo. An extensive study on LES, RANS and hybrid RANS/LES simulation of a narrow-gap open Taylor-Couette flow. 9th Symposium on DNS, LES, and Hybrid RANS/LES Methods, Aug 2014, Chicago, United States. hal-01098588

\section{HAL Id: hal-01098588 https://hal.science/hal-01098588}

Submitted on 8 Jan 2015

HAL is a multi-disciplinary open access archive for the deposit and dissemination of scientific research documents, whether they are published or not. The documents may come from teaching and research institutions in France or abroad, or from public or private research centers.
L'archive ouverte pluridisciplinaire HAL, est destinée au dépôt et à la diffusion de documents scientifiques de niveau recherche, publiés ou non, émanant des établissements d'enseignement et de recherche français ou étrangers, des laboratoires publics ou privés. 
FEDSM2014-21335

\title{
AN EXTENSIVE STUDY ON LES, RANS AND HYBRID RANS/LES SIMULATION OF A NARROW-GAP OPEN TAYLOR-COUETTE FLOW
}

\author{
Christophe Friess; Sébastien Poncet \& Stéphane Viazzo \\ Aix-Marseille Université, CNRS, Ecole Centrale \\ Laboratoire M2P2, UMR 7340 \\ 38, rue Frédéric Joliot-Curie \\ 13451 MARSEILLE Cedex 13 \\ friess@|3m.univ-mrs.fr
}

\begin{abstract}
The present paper concerns a numerical benchmark of various turbulence modelings, from RANS to LES, applied to Taylor-Couette-Poiseuille flows in a narrow gap cavity for six different combinations of rotational and axial Reynolds numbers. Two sets of refined Large-Eddy Simulation results, using the WALE and the Dynamic Smagorinsky subgrid scale models available within an in-house code based on high-order compact schemes, hold for reference data. The efficiency of a RANS model, the Elliptic Blending Reynolds Stress Model (EB-RSM) [1], and a hybrid RANS/LES method, the so-called "Equivalent DES" [2], both run with Code Saturne, is then questioned. Thin coherent structures appearing as negative (resp. positive) spiral rolls are obtained by the LES but also the hybrid RANS/LES along the rotor (resp. stator) sides. More quantitatively, the hybrid RANS/LES does not improve the predictions of the EBRSM for both the mean and turbulent fields, stressing the need for further theoretical development.
\end{abstract}

\section{NOMENCLATURE}

$C, C_{m}$ model parameters.

$C_{\varepsilon 1}, C_{\varepsilon 2} \quad$ model parameters.

$\delta t$ time step.

$h$ length of the cylinders.

$k$ total (resolved+modeled) turbulence kinetic energy.

$k_{m}$ modeled part of the turbulence kinetic energy.
$L \quad$ length scale.

$N$ rotation parameter, $N=R e_{\Omega} / R e_{Q}$.

$r$ radial coordinate.

$r_{k}$ energy ratio $r_{k}=k_{m} / k$.

$\operatorname{Re}_{Q} \quad$ axial Reynolds number, $\operatorname{Re}_{Q}=W_{m}\left(R_{2}-R_{1}\right) / v$.

$R e_{\Omega}$ rotational Reynolds number, $R_{\Omega}=\Omega R_{1}\left(R_{2}-R_{1}\right) / v$.

$R_{1}, R_{2}$ radii of the inner and outer cylinders respectively.

$R_{i j} \quad$ Reynolds stress tensor components.

$S_{i j} \quad$ Strain-rate tensor components.

$U$ Velocity.

$V_{\theta}, V_{z}$ mean tangential and axial velocity components.

$W_{m}$ bulk inlet axial velocity.

$z$ axial coordinate.

\section{Subscripts and superscripts}

$c$ cutoff.

$d$ deviatoric.

$i, j \quad$ indices for tensors; $(i, j)=(r, \theta, z)$.

$m$ modeled.

$s$ sweeping.

filtered quantity.

SFS subfilter-scale.

$t$ turbulent.

Greek symbols

$\alpha$ parameter of the EB-RSM.

$\beta_{0}$ model constant.

$\Gamma$ aspect ratio of the cavity, $\Gamma=h /\left(R_{2}-R_{1}\right)$.

$\Delta$ grid step.

$\varepsilon \quad$ dissipation rate $\varepsilon=\varepsilon_{k k} / 2$. 
$\varepsilon_{i j} \quad$ dissipation rate tensor components.

$\eta$ radius ratio, $\eta=R_{1} / R_{2}$.

$v$ fluid kinematic viscosity.

$v_{t}$ turbulent eddy viscosity.

$\phi_{i j}^{*} \quad$ redistribution tensor components.

$\theta$ azimuthal coordinate.

$\omega$ pulsation.

$\Omega$ rotation rate.

\section{Acronyms}

DES Detached-Eddy Simulation.

EB-RSM Elliptic Blending Reynolds Stress Model.

LES Large-Eddy Simulation.

RANS Reynolds-Averaged Navier-Stokes.

WALE Wall-Adapting Local Eddy Viscosity.

\section{INTRODUCTION}

Since Taylor's first theoretical results obtained one century ago, huge research efforts have been done to better describe and understand the rich dynamical behavior exhibited by the flow induced by the differential rotation of two concentric cylinders. This kind of flow has found many applications in chemical engineering or in the turbomachinery industry for examples. In the present case, the main motivation arises from the cooling of electrical motors, which can be modeled quite faithfully by considering a narrow-gap Taylor-Couette system with an axial Poiseuille flow. A better knowledge of the hydrodynamic field is absolutely necessary before considering the heat transfer aspect as pointed out recently by Fénot et al. [3]. These last authors provided a very useful and exhaustive literature survey on Taylor-Couette flows with or without an axial Poiseuille flow and including or not heat transfer processes. They explained the difficulty to establish universal correlations for the heat transfer coefficients by the large numbers of parameters involved in the problem. Moreover, most of the previous studies were mainly concerned with temperature measurements without any idea of what the hydrodynamic flow was and especially what the inlet flow conditions were. In the present work, one proposes a numerical benchmark for various combinations of the flow parameters in the isothermal case. It is a step forward the turbulence modeling of the full problem with heat transfer.

Nouri and Whitelaw [4] then Escudier and Gouldson [5] provided very useful experimental databases for middle-gap cavities $(\eta \simeq 0.5)$ of large aspect ratios $(\Gamma=98$ and 244 respectively). Until now, most numerical studies used turbulence modeling, which provided rather limited informations. It was shown by $[6,7]$ that increasing the rotation rate of the inner cylinder amplified the turbulence kinetic energy, resulting in the enhancement of heat transfer along the rotor. More recently, the development of computational methods (including Direct and Large Eddy Simulations) has led to an increase in numerical studies of rotating flows, but few works were concerned by turbulent
Taylor-Couette-Poiseuille flows and especially the near-wall turbulent structures in such systems. As example, in the middlegap configuration, Chung and Sung [8] established the destabilization of the near-wall turbulent structures due to rotation of the inner wall giving rise to an increase of sweep and ejection events. In the same way, Hadziabdic et al. [9] studied by LES a fully-developed turbulent flow in a concentric annulus of radius ratio $\eta=0.5$, with the outer wall rotating at a range of rotation rates $N=R e_{\Omega} / R e_{Q}$ from 0.5 up to 4 . They focused their attention on the effect of the rotation parameter $N$ on the turbulence statistics and coherent structures in the near-wall regions. To our knowledge, there is no reference numerical or experimental data for the narrow-gap case $(\eta>0.8)$. Thus, the present in-house code based on fourth-order compact schemes using the dynamic Smagorinsky subgrid scale model has been first validated by Oguic et al. [10] against the experimental data of Nouri and Whitelaw [4]. Its slightly improved the LES results of Chung and Sung [8] based on second-order numerical schemes highlighting the importance of the order of the spatial schemes. It will be considered here as providing the reference data to discuss the capability of RANS and hybrid RANS/LES of predicting the mean and turbulent flow fields in the narrow-gap case.

The present work is an extension of Friess et al. [11] to more combinations of the rotational and axial Reynolds numbers and to more turbulence modelings. Its goal remains twofold: (i) providing some reference LES data using two subgrid scale modes available within an in-house high-order solver and (ii) questioning the capabilities of a hybrid RANS / LES method, as well as the underlying RANS model, in predicting this kind of flow. The paper is organized as follows: the flow configuration is first described. The numerical approaches are then presented. Afterwards, the results about the hydrodynamic fields are discussed in details in terms of the coherent structures and the mean and turbulent flow fields, before some final conclusions.

\section{FLOW CONFIGURATION AND PARAMETERS}

The fluid is confined between two concentric cylinders of radii $R_{1}$ and $R_{2}$ and height $h$ (see Figure 1 ). The inner cylinder rotates at a constant rate $\Omega$, while the outer cylinder is stationary. The cavity is characterized by two geometrical parameters : its aspect ratio $\Gamma=h /\left(R_{2}-R_{1}\right)$ and its radius ratio $\eta=R_{1} / R_{2}$. An axial throughflow is imposed within the gap at a constant bulk velocity $W_{m}$. The main flow parameters are the rotational Reynolds number $\operatorname{Re}_{\Omega}=\Omega R_{1}\left(R_{2}-R_{1}\right) / v$ and the bulk Reynolds number $\operatorname{Re}_{Q}=W_{m}\left(R_{2}-R_{1}\right) / v, v$ being the fluid kinematic viscosity. The present study considers two values of the axial flow rate and three rotation rates of the inner cylinder, resulting in five distinct values of $N=\operatorname{Re}_{\Omega} / \operatorname{Re}_{Q}$ (see Table 1$)$. 


\begin{tabular}{|c|c|c|c|c|c|c|}
\hline Case & $\mathrm{A}$ & $\mathrm{B}$ & $\mathrm{C}$ & $\mathrm{D}$ & $\mathrm{E}$ & $\mathrm{F}$ \\
\hline$\Gamma$ & \multicolumn{6}{|c|}{10} \\
\hline$\eta$ & \multicolumn{7}{|c|}{$8 / 9$} \\
\hline$R e_{Q}$ & \multicolumn{7}{|c|}{3745} & \multicolumn{3}{c|}{5617} \\
\hline$R e_{\Omega}$ & 8378 & 16755 & 25133 & 8378 & 16755 & 25133 \\
\hline$N$ & 2.24 & 4.47 & 6.71 & 1.49 & 2.98 & 4.47 \\
\hline
\end{tabular}

TABLE 1. Geometrical and flow parameters

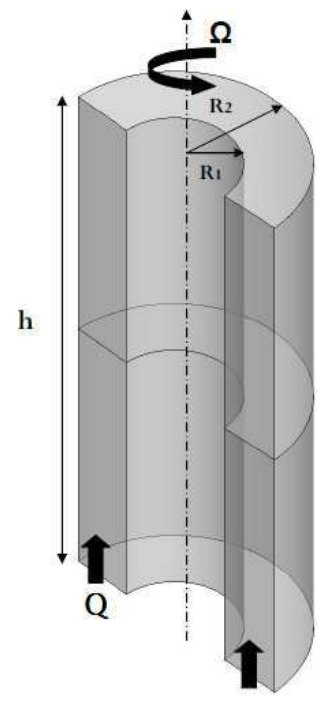

FIGURE 1. Sketch of the narrow-gap cavity

\section{NUMERICAL METHODS}

Concerning LES reference calculations, the numerical method of the in-house code is based on the former work of Abide and Viazzo [12], who devised a 2D compact fourth-order projection decomposition method in cartesian coordinates. It has been recently validated in the case of turbulent interdisk rotorstator flows in cylindrical coordinates by Viazzo et al. [13] but also for turbulent Taylor-Couette-Poiseuille flows in a middlegap cavity by Oguic et al. [10]. The time advancement is secondorder accurate and is based on the explicit Adams-Bashforth scheme for the convective terms and an implicit backward Euler scheme for the viscous terms. The derivatives are approximated using fourth-order compact formula in the radial and axial directions. The time splitting scheme is an improved projection method, which ensures the incompressibility at each time step. The projection decomposition method is based on a direct nonoverlapping multidomain Helmholtz/Poisson solver, which provides the solution of each Helmholtz/Poisson problem resulting of the velocity-pressure coupling. The multidomain solver ensures the continuity of the solution and its first normal derivative across the conforming interface. These continuity conditions are cleared up using an influence matrix technique. By assuming the periodicity of the solution in the azimuthal direction, the threedimensional extension uses Fourier series. The set of problems is thus reduced to series of two-dimensional problems associated with each wave number. Periodic boundary conditions are applied in the axial and circumferential (angle of $3 \pi / 4$ ) directions and no-slip boundary conditions are imposed on the walls. The cavity is divided into 2 subdomains in the axial direction. All the numerical details including the mesh grid, the time step and the wall coordinates are provided in Table 2 .

The CFD code used for RANS and hybrid RANS/LES simulations is the open-source Code_Saturne, developed by EDF [14]. It is a finite volume solver, written in cartesian coordinates. A SIMPLEC algorithm, with the Rhie and Chow interpolation, is used for pressure-velocity coupling. Concerning hybrid RANS/LES calculations, convective fluxes are approximated by a second-order centered scheme, for momentum, and a first-order upwind scheme for subfilter quantities. Time marching uses a Crank-Nicholson, second-order scheme. The hybrid RANS/LES mesh contains $60^{3}$ cells, with an angular periodicity of $\pi / 2$ and an axial periodicity. No-slip boundary conditions are imposed on the walls. The RANS mesh is a $1 \mathrm{D}$ grid of 60 cells. In RANS mode, convective fluxes are approximated by a first-order upwind scheme, for all computed quantities, and the time scheme is first-order.

\section{TURBULENCE MODELLING}

Various levels of modeling are presented. Emphasis is first put on the hybrid RANS/LES method. Afterwards, the subfilter closure and RANS model will be briefly presented.

\section{Hybrid Method}

The hybrid method used for the present work is the "Equivalent DES" of Friess and Manceau [15] and Manceau et al. [2]. This approach was first derived for the purpose of bridging the PITM (Partially Integrated Transport Model) [16] and the DES (Detached Eddy Simulation) method. Indeed, the first is fully justified from a theoretical point of view, while the second one was developed on a rather phenomenological basis. The PITM was first generalized by Fadai-Ghotbi et al. [17] to inhomogeneous flows, considering temporal filtering, rather than spatial filtering. From a pragmatical point of view, considering RANS / TLES (temporal LES) hybridization does not cause any difficulty in implementing models or special terms, since Fadai-Ghotbi et al. showed that applying Temporal PITM (T-PITM) to an inhomogeneous stationary flow is equivalent to applying PITM to homogeneous, statistically unsteady flow. Actually, one must just keep in mind that "hybrid RANS/LES" is to be understood in a general way, including temporal LES. 
The advantage of Detached Eddy Simulation lies in its simplicity and robustness. The idea of Manceau et al. [15] was thus to derive an approach bridging it with T-PITM. This latter is, in spite of its theoretical justification, not very easy to implement in any code. An equivalence criterion was then determined between T-PITM and DES, providing some theoretical justification to the latter, and allowing to interpret it as a hybrid RANS / TLES method. This equivalence criterion was derived analytically for equilibrium flows, but was successfully tested on a flow over a periodic hill, involving massive separation [2].

The principle of DES is to magnify the dissipation term of the transport equation for either subfilter turbulence kinetic energy $k_{S F S}$ or subfilter stresses $\tau_{i j S F S}$, damping the modeled energy and allowing large scale eddies to be resolved:

$$
\varepsilon_{i j}^{D E S}=\frac{k_{S F S}^{3 / 2}}{\varepsilon_{S F S} L} \varepsilon_{i j S F S}
$$

While classical DES uses the local grid step to determine the length scale $L$ in Eq.(1), equivalent DES uses the ratio $r_{k}$ defined as: $r_{k}=k_{m} / k$, where $k$ is the total turbulence kinetic energy and $k_{m}$ its counterpart contained in the modeled (subfilter) scales. At the RANS limit, $r$ tends to 1 , and at the DNS limit, it tends to 0 . The advantage of $r_{k}$ is that it can be estimated using a spatial or a temporal energy spectrum. As shown by Friess and Manceau [2], $r_{k}$ can be evaluated as:

$$
\begin{aligned}
r_{k} & =\frac{1}{k} \int_{\omega_{c}}^{\infty} E_{T}(\omega) d \omega \\
& =\min \left(1, \frac{1}{\beta_{0}}\left(\frac{U_{s}}{\sqrt{k}}\right)^{2 / 3}\left(\omega_{c} \frac{k}{\varepsilon}\right)^{-2 / 3}\right)
\end{aligned}
$$

$\beta_{0}$ being a constant derived from the Kolmogorov constant $\left(\beta_{0}=\right.$ 0.3 in the present case), $U_{S}$ a sweeping velocity and $\omega_{c}$ the cutoff frequency of the considered filter. It can be defined as :

$$
\omega_{c}=\min \left(\frac{\pi}{\delta t} ; \frac{U_{s} \pi}{\Delta}\right)
$$

where $\delta t$ and $\Delta$ are the time and grid steps, respectively. Finally, following [15], the length scale $L$ entering Eq.(1) is :

$$
L=\frac{r_{k}^{3 / 2}}{1+\frac{C_{\varepsilon 2}-C_{\varepsilon 1}}{C_{\varepsilon 1}}\left(1-r^{C_{\varepsilon 1} / C_{\varepsilon 2}}\right)} \frac{k^{3 / 2}}{\varepsilon}
$$

\section{RANS model and subfilter closure}

In the temporally-filtered Navier-Stokes equations, the subfilter stresses must be modeled such a way that, making the temporal filter width go to infinity, the equations tend to the RANS equations. In particular, one of the main objectives of hybrid methods is to use RANS closures in the near-wall regions, to avoid the very fine resolution required by LES. In that aim, the RANS model proposed by Manceau and Hanjalic [1] and Manceau [18], the so-called elliptic blending Reynolds-Stress Model (EB-RSM), is adapted to the hybrid temporal LES context. In this model, an elliptic relaxation equation is solved for a scalar $\alpha$ :

$$
\alpha-L_{S F S}^{2} \nabla^{2} \alpha=1
$$

which is a sensor of the distance to the wall $(\alpha=0$ at the wall, and 1 far away), and is used to blend near-wall and homogeneous formulations for the redistribution and dissipation terms of the transport equations for subfilter stresses (see [19] for details) :

$$
\phi_{i j}^{*}-\varepsilon_{i j}=\alpha^{3}\left(\phi_{i j}^{*}-\varepsilon_{i j}\right)_{\mathrm{wall}}+\left(1-\alpha^{3}\right)\left(\phi_{i j}^{*}-\varepsilon_{i j}\right)_{\text {homogeneous }} \text {. }
$$

Under its RANS form, EB-RSM was successfully applied to several flows, e.g. non-rotating and rotating channels [18], and mixed and natural convection flows [20] among other things. It was recently adapted by Fadai-Ghotbi et $a l$. [19] to serve as a subfilter-stress model in the framework of T-PITM. In the present work, it is also applied as a model for the equivalent DES, by simply substituting the dissipation in the fashion of Eq. (1) in the Reynolds-stress transport equations.

\section{Subgrid-Scale Models}

Reference data are here provided by Large Eddy Simulations, using two different subgrid-scale models implemented in the in-house code based on fourth-order compact schemes previously described. The Wall-Adapting Local Eddy Viscosity (WALE) model of Nicoud and Ducros [21] has already been considered by Friess et $a l$. [11] in the same configuration for particular values of the rotation parameter $N$. The study is extended to other values of $N$ and to the use of the Dynamic Smagorinsky model developed by Germano et al. [22].

The Dynamic Smagorinsky model In the Smagorinsky model, the eddy viscosity is assumed to be proportional to the subgrid scale characteristic length $\Delta=(r \Delta r \Delta \theta \Delta z)^{1 / 3}$ and to a characteristic turbulent velocity taken as the local strain rate $|\bar{S}|=\sqrt{2 \bar{S}_{i j} \bar{S}_{i j}}$ :

$$
v_{t}=\left(C_{S} \Delta\right)^{2}|\bar{S}|
$$

with $C_{S}=0.2$. The classical Smagorinsky model appeared to be too dissipative in a previous work on turbulent rotor-stator 
flows [13]. That is the reason why one uses it in its dynamical version, where $C_{s}$ is replaced by $C_{d}$, which depends on both space and time. $C_{d}$ is evaluated with a least-squares approach as a part of the solution at each time step using a test filter equal to $2 \Delta$. $C_{d}$ is then averaged along the tangential direction (see in [22,23]).

The WALE model This choice is motivated by a better numerical stability than the dynamic Smagorinsky model, and its quality in near-wall treatment without damping functions. The subgrid viscosity is given by :

$$
v_{t}=\left(C_{m} \Delta\right)^{2} \frac{\left(S_{i j}^{d} S_{i j}^{d}\right)^{3 / 2}}{\left(\bar{S}_{i j} \bar{S}_{i j}\right)^{5 / 2}+\left(S_{i j}^{d} S_{i j}^{d}\right)^{5 / 4}}
$$

where $C_{m}^{2}=10.6 C_{s}^{2}=0.424$ is constant, $\Delta$ the grid step, $\bar{S}_{i j}$ the filtered strain-rate tensor, and $S_{i j}^{d}$ the deviatoric part of the squared filtered velocity gradient tensor. For the same mesh grid, the WALE model enables to save around $12 \%$ of the computational cost compared to the dynamic Smagorinsky model as shown in Table 2 mainly because it does not use any test filtering procedure.

\section{RESULTS AND DISCUSSION}

A new experimental set-up has been recently designed at IRPHE for the same sets of parameters $\left(\eta=0.89, R_{\Omega}\right.$ and $R e_{Q}$ values) but for a relatively small aspect ratio $\Gamma=50$, such that the hydrodynamic flow is never established whatever the values of the axial flow and rotation rates (see extensive comparisons in [24]). As the in-house code has already been validated for a middle-gap cavity [10], it will hold for reference data in the following.

From Figures 2 to 5, a strong agreement between the WALE and the Dynamic Smagorinsky models, is obtained whatever the value of the rotation parameter. The profiles are indistinguishable most of the time with slightly higher intensity levels in the core of the flow provided by the WALE model. For the same mesh grid and so the same wall coordinates, the Dynamic Smagorinsky model provides slightly higher values of the friction Reynolds numbers along both walls. Both LES will be used in the following as reference data for comparisons between the RANS and the hybrid RANS/LES approaches. One can recall that the WALE enables to save about $12 \%$ of computational cost compared to the Dynamic Smagorinsky model (see Table 2).

The choice of the rotation parameter $N=R e_{\Omega} / R e_{Q}$, as the main parameter governing the hydrodynamics of Taylor-CouettePoiseuille flows, is first discussed. Indeed, cases B and F have the same value of $N=4.47$, though different rotation and axial flow rates. The same normalization as used by Chung and Sung [8] is introduced: $\Omega R_{1}$ for the azimuthal mean and root mean square velocities, and $W_{m}\left(\right.$ or $\left.W_{m}^{2}\right)$ for other quantities. Figure 2 shows the first and second moments, exhibiting no significant differences between those two cases, whether modeling approach (LES, hybrid RANS/LES or RANS) is considered. It confirms that the rotation parameter pilots the hydrodynamics in such open Taylor-Couette system for this range of $N$ values $[1.49-6.71]$. One can expect that rotation effects dominate compared to those of the axial flow as $N$ remains larger than unity.
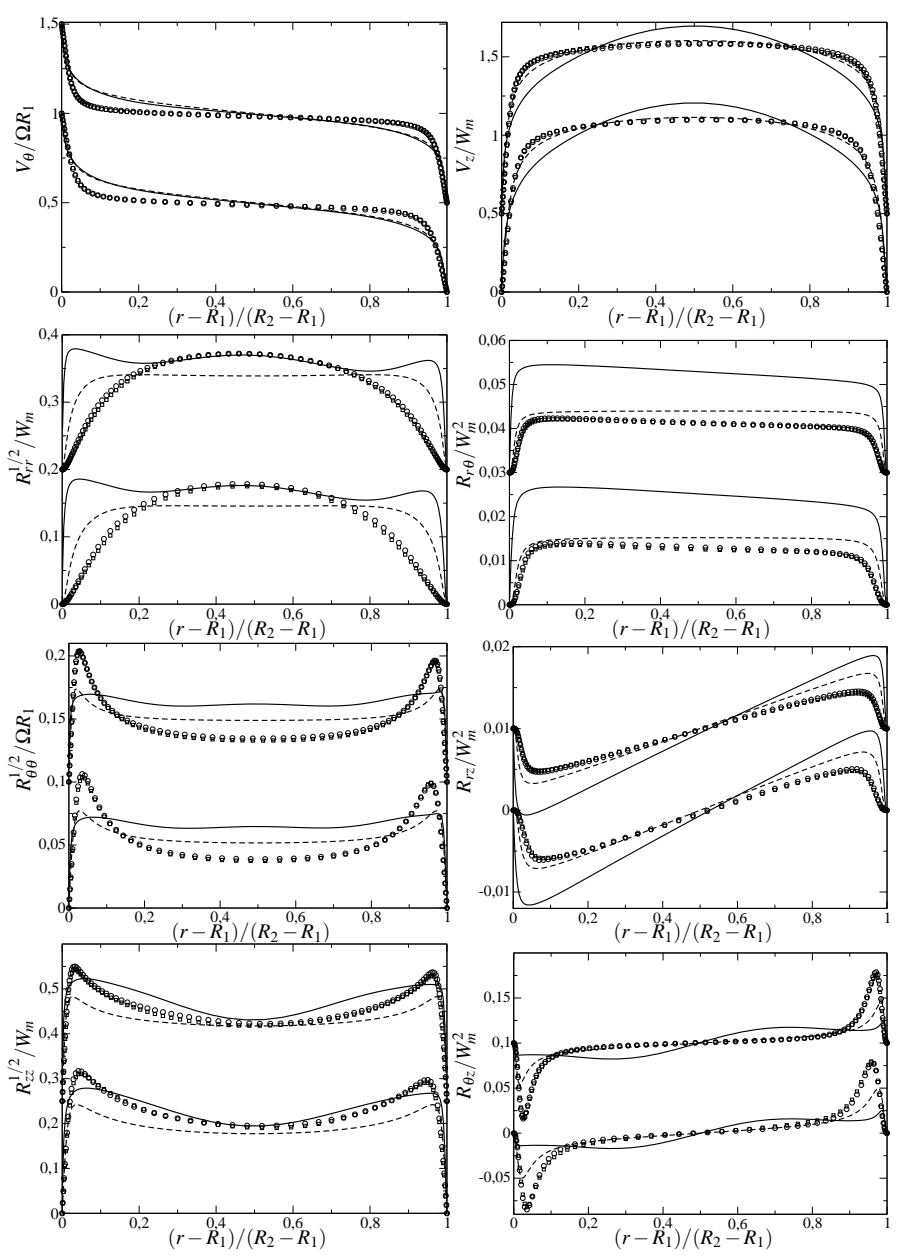

FIGURE 2. Azimuthal and axial velocity components and all six Reynolds stress tensor components for $N=4.47$. Cases F (shifted up) and B. $\square$ : Dynamic Smagorinsky model, ० : WALE model, —- : hybrid RANS/LES, -- : RANS.

Figs. 3 through 5 compare all four other cases, put two by two on the same graphs, such as the left side is for $N=1.49$ (case D) and $N=2.24$ (case A), while the right side shows profiles for $N=2.98$ (case E) and $N=6.71$ (case C). The LES provide rather the same mean velocity distributions whatever the value of $N$. The LES predict a mean flow divided into three flow regions: 
two thin boundary layers on each wall separated by a central core at mid-gap in almost solid body rotation. For $N=1.49$, the mean tangential velocity slightly decreases with the radius at mid-gap, highlighting weak turbulence intensities. For larger values of $N$, the slope of $\partial\left(V_{\theta} / \Omega R_{1}\right) / \partial r$ in the free stream, asymptotically tends to 0 , such that $V_{\theta}$ gets constant, which is typical of turbulent regimes in rotating flow configurations. It may be explained by considering an effective Reynolds number based on the effective velocity $V_{e f f}=\sqrt{W_{m}^{2}+\beta\left(\Omega R_{1}\right)^{2}}$ ( $\beta$ a constant generally put to 0.5$)$. At a given axial flow rate, increasing the rotation parameter leads to an increase in the effective velocity or Reynolds number and as a consequence to higher turbulence levels. Furthermore, the axial dimensionless velocity $V_{z}$ is also weakly influenced by $N$, at least concerning LES and RANS calculations. This latter behavior is different from that of middle-gap $(\eta \simeq 0.5)$ Taylor-Couette-Poiseuille flows, for comparable values of $N$. For example, Hadziabdic et al. [9] have shown that increasing $N$ puts the axial velocity towards a laminar Poiseuille-like shape. Here, the radial distribution of $V_{z}$ is typical of what is encountered in turbulent pipe flows regarding the LES and RANS profiles. On the other hand, the hybrid RANS/LES approach exhibits larger radial variations with a profile similar to a laminar Poiseuille-like profile indicating lower turbulence intensities at mid-gap.
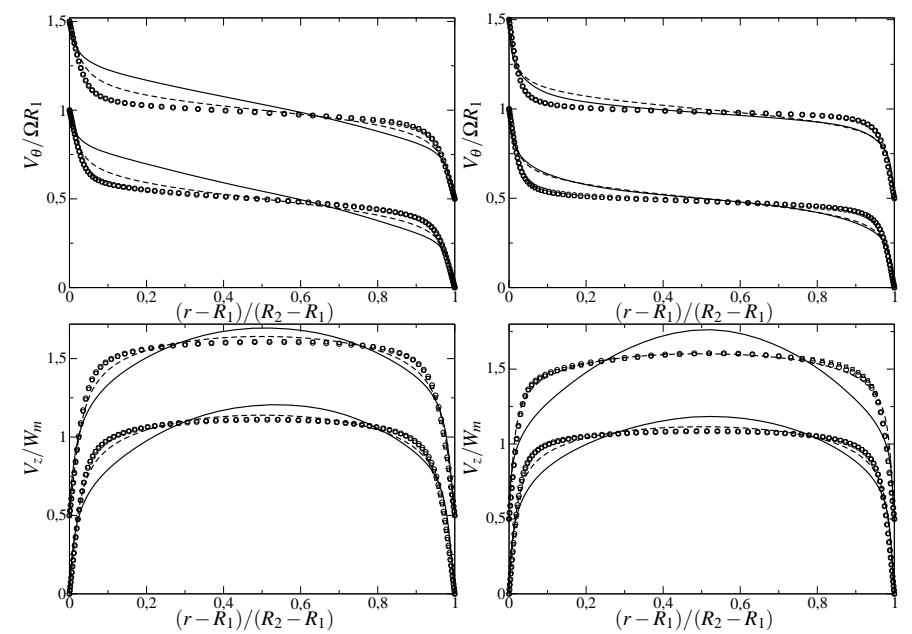

FIGURE 3. Azimuthal (top) and axial (bottom) mean velocities. Left : cases A (shifted up) and D. Right : cases C (shifted up) and E. Same legend as Figure 2.

Regarding the radial distributions of all six Reynolds stress tensor components obtained by LES (Fig.4 and 5), turbulence is mainly concentrated in the thin boundary layers developed along the walls with remarkably high peak values. All profiles in absolute values are slightly asymmetric. The rotor boundary layer appears besides to be more unstable than those of the stator high- lighting the destabilizing effect of rotation for this range of $N$ values. The normal components $R_{\theta \theta}$ and $R_{z z}$ are twice lower in the core region. The shear stress components $R_{r z}$ and $R_{\theta z}$ show strong stresses along both walls. Increasing the value of $N$ leads to an increase of the turbulence intensities in all flow regions.
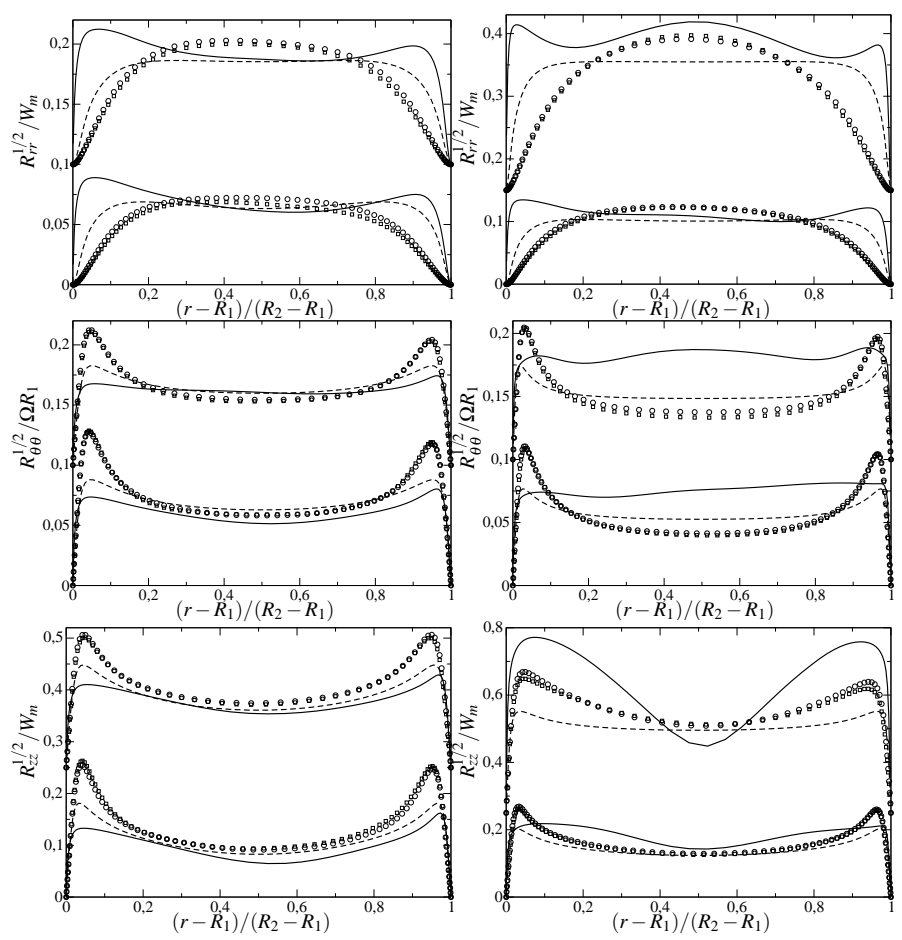

FIGURE 4. Normal components of the Reynolds stress tensor. See legend of Figure 3.

The prediction capabilities of both RANS and hybrid RANS/LES approaches used here, are now discussed. Both approaches provide roughly the good profiles with larger discrepancies for some components of the Reynolds stress tensor. Clearly, the hybrid RANS/LES approach does not improve the predictions of the RANS model, which behaves quite well. The EBRSM model has previously shown some satisfactory results on channel flows with or without global rotation (see e.g. [18]). But in its hybridization, under the Equivalent DES method, it shows heterogeneous prediction quality, with strong dependence on $N$. Generally, they both overestimate the peak intensities for the $R_{r r}$ and cross components. Thus, they also overpredict the values of the friction Reynolds number as shown in Table 2.

For instance, for case $\mathrm{D}(N=1.49)$, the profile of the energy ratio $r_{k}=k_{m} / k$, shown by Figure 6 , betrays an unexpected RANS state, which can even be assimilated to a relaminarization, according to the azimuthal velocity profile (Figure 3 ). This weakness can be compared to the difficulty of any DES-like ap- 

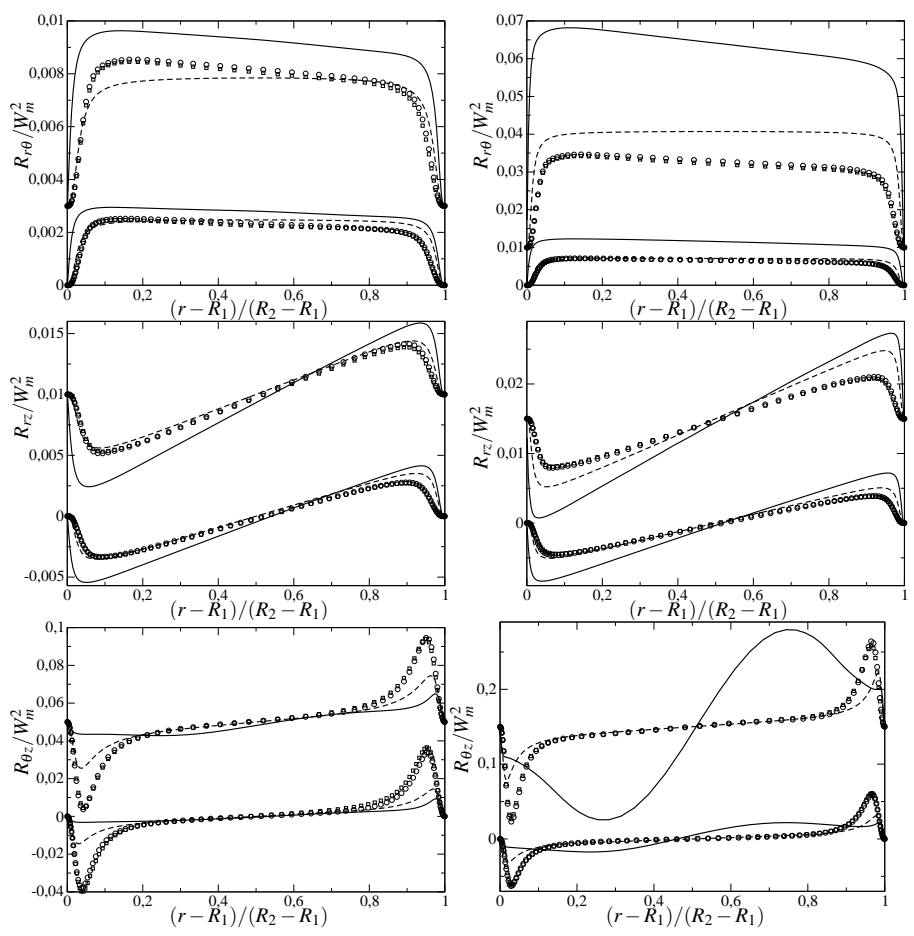

FIGURE 5. Shear stress components of the Reynolds stress tensor. See legend of Figure 3.

proach, to sustain a fluctuating state in quite stable configurations. Even case A $(N=2.24)$ shows a similar tendency. For higher values of $N$, statistics of the flow seem to be better predicted, however with flat profiles for the highest value (case $\mathrm{C}$, $N=6.71$ ). Indeed, while Figure 6 shows that the energy ratio $r_{k}$ is well driven in the free flow, second moment profiles (see Figs.5 and 4) seem to exhibit which could be considered as unphysical resolved structures. This would be the result of an unsufficient dissipation rate, thus suggesting that the value of $\beta_{0}$ (see Eq.(2)) should be lowered.

On the fringe of the previous observations, one can have a look upon the isovalues of the Q-criterion shown on Figures 7 and 8. The isovalues of the Q-criterion obtained by the LESWALE are shown on the rotor and stator sides, while for the hybrid RANS/LES, the isovalues are shown only in front of the rotor, since there are far less coherent structures on the stationary cylinder. The LES highlights the presence of 3D unsteady coherent structures within the two boundary layers. They appear as thin negative spiral patterns along the rotor as they roll up in the opposite sense of the inner cylinder rotation. The same spiral network is obtained along the stator with a positive angle. When the axial throughflow is more important (weak values of $N$ ), these structures are more aligned with the axial direction. They get progressively inclined with the tangential direction when the rotation rate increases. As examples, along the rotor, the angle

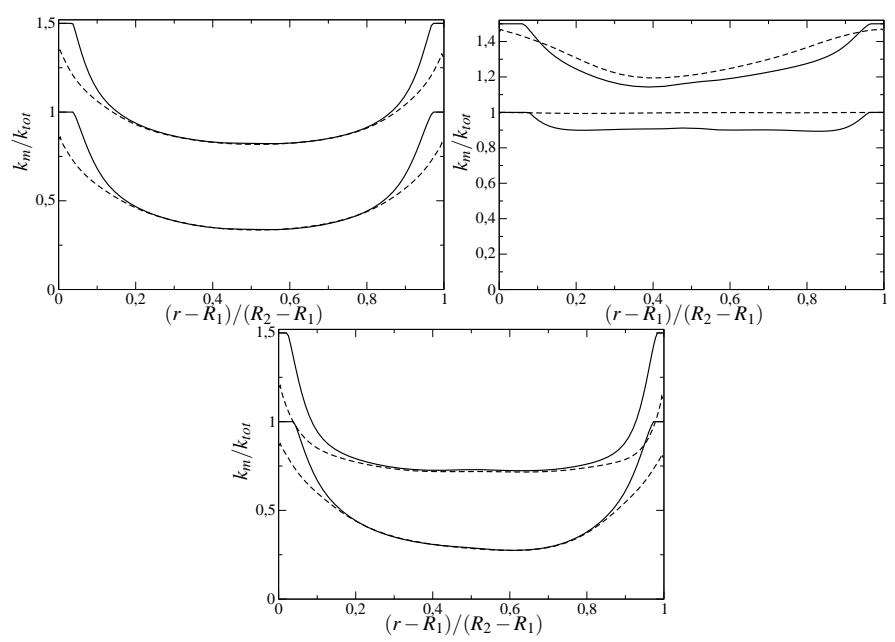

FIGURE 6. Energy ratio $r=k_{m} / k_{\text {tot }}$. Left : cases F (shifted up) and B. Right : cases A (shifted up) and D. Bottom : cases C (shifted up) and E. - : : targetted, -- observed.

formed by the spirals with the tangential direction is equal to $-45^{\circ}$ for $N=1.49$ and to $-16^{\circ}$ for $N=6.71$. Despite the hybrid approach captures less structures, these spiral rolls form rather the same angle with the tangential direction as in the LES, except for case $\mathrm{C}$, where the streaks captured by the hybrid RANS/LES are almost horizontal. These 3D unsteady structures appearing as thin spiral rolls along both walls at a relatively small scale in the LES are responsible for the large peak values observed in the Reynolds stress tensor profiles. They may play a key role in the wall heat transfer process. They could explain why most of the RANS models, which assume the base flow as being stationary and axisymmetric, fail to predict the right distributions of the heat transfer coefficient.

Finally, Table 2 contained in Appendix A, shows some numerical parameters, as well as wall quantities, for all six cases, and for all four approaches (both LES, hybrid RANS/LES and RANS). Globally, this table confirms that the RANS approach provides satisfactory results for these quantities. Another observation that can be made, is that the friction Reynolds numbers are poorly predicted by the hybrid approach on both walls. This may be linked to the fact that the RANS mode $\left(r_{k}=1\right)$ is not well enforced on either wall (Figure 6).

\section{CONCLUDING REMARKS}

The present work is a further step for Friess et al. [11], on six different values of the rotation parameter $N$ providing a wider numerical data set of Taylor-Couette-Poiseuille flows in a narrow-gap rotor-stator cavity $(\eta=0.89)$. Since the LES code was validated in a middle-gap $(\eta=0.5)$ configuration [10], and since there is no available experimental data set for a fully established narrow-gap flow (see e.g. [24]), its results may be con- 

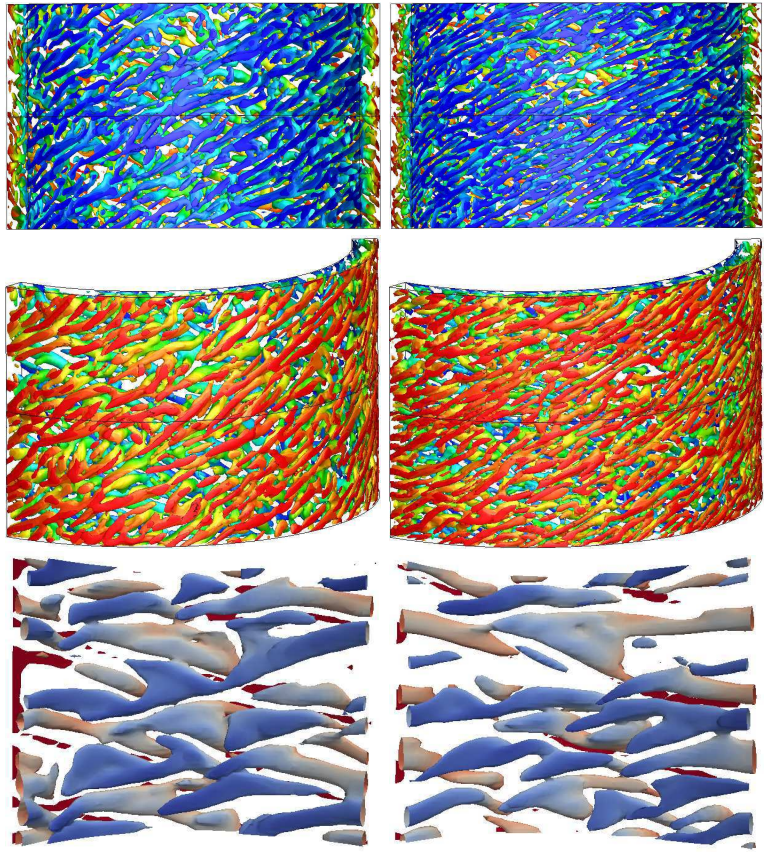

FIGURE 7. Isocontours of the Q-criterion in the full computational domain, colored by the radial coordinate $r$ (blue on the rotor and red on the stator). Left : Case B, right : case F. From top to bottom : LESWALE, respectively rotor and stator side, and hybrid RANS/LES, rotor side. The inner cylinder is rotating counter-clockwise and the axial throughflow is from the bottom to the top.

sidered as reference. One used here two subgrid scale models, the Dynamic Smagorinsky and WALE models, providing very similar results for both the mean and turbulent fields, while the WALE enables to save about $12 \%$ of computational effort. The $N$ values are in the range $[1.49 ; 6.71]$, such that a parametric study can be performed. Three observations may be put forth: (i) as for middle-gap Taylor-Couette-Poiseuille flows, $N$ is the main parameter governing the flow, (ii) the dimensionless azimuthal velocity slightly varies with $N$, (iii) unlike middle-gap TaylorCouette-Poiseuille flows, the axial velocity profile does not significantly vary with $N$, even for high $N$ values.

The conclusions about the performance of the so-called "Equivalent DES" hybrid RANS/LES approach, are as follows: (i) no significant improvement in predicting flow statistics, by comparison with the underlying RANS (EB-RSM) model, but (ii) Q-criterion isocontours exhibit net-shaped streaks, as well as the changing of the angle of the main directions of these structures, in relatively good accordance with the reference LES.

Eventually, one can say that this work is a new illustration of the fact that seamless hybrid RANS/LES methods are better suited for flows far from equilibrium, for instance involving separation. However, further theoretical work on "grey zones" treatment, could shake things up.

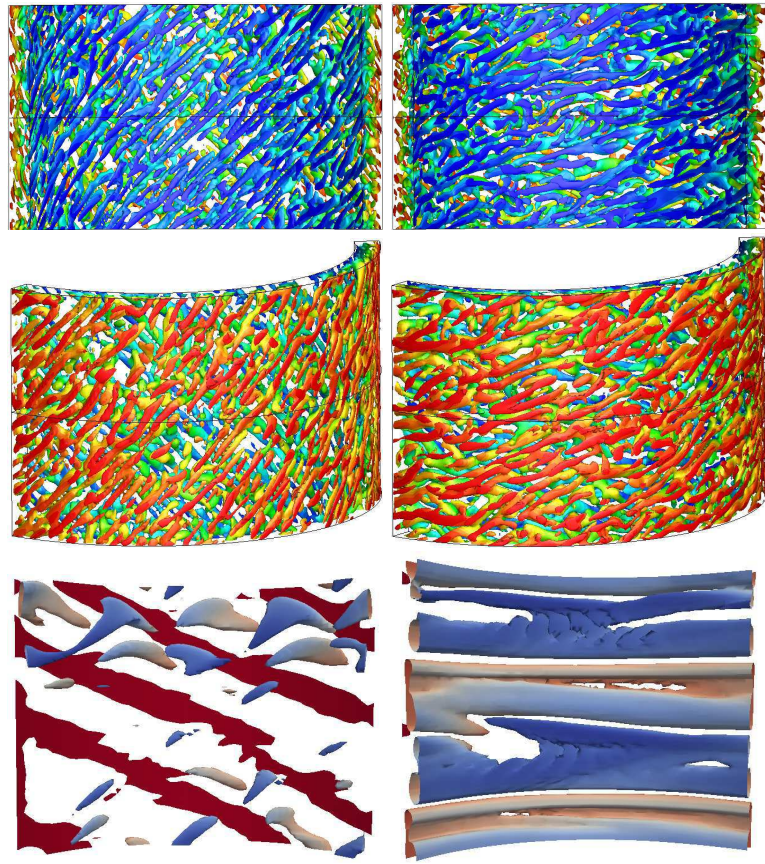

FIGURE 8. Isocontours of the Q-criterion, colored by the radial coordinate $r$. Left : Case A, right : case C. Same legend as Figure 7.

\section{ACKNOWLEDGMENT}

This work was financially supported by Liebherr Aerospace (project "Entrefer moteur") and by the LABEX MEC (ANR-11LABX-0092) through the HYDREX program, which are gratefully acknowledged. Furthermore, this work was granted access to the HPC resources of IDRIS under the allocation 2013-090242 made by GENCI (Grand Equipement National de Calcul Intensif). 


\section{REFERENCES}

[1] Manceau, R., and Hanjalić, K., 2002. "Elliptic blending model: A new near-wall Reynolds-stress turbulence closure". Phys. Fluids, 14(2), pp. 744-754.

[2] Friess, C., and Manceau, R., 2012. "Investigation of the equivalence of two hybrid temporal-LES methods based on elliptic blending in separated flows". In Proc. 7th Int. Symp. Turbulence, Heat and Mass Transfer, Palermo.

[3] Fénot, M., Bertin, Y., Dorignac, E., and Lalizel, G., 2011. "A review of heat transfer between concentric rotating cylinders with or without axial flow". Int. J. Thermal Sci., 50(7), pp. 1138-1155.

[4] Nouri, J. M., and Whitelaw, J. H., 1994. "Flow of Newtonian and non-Newtonian fluids in a concentric annulus with rotation of the inner cylinder". J. Fluid. Eng., 116, pp. 821-827.

[5] Escudier, M., and Gouldson, I., 1995. "Concentric annular flow with centerbody rotation of a newtonian and a shearthinning liquid". Int. J. Heat Fluid Flow, 16, pp. 156-162.

[6] Poncet, S., Haddadi, S., and Viazzo, S., 2011. "Numerical modeling of fluid flow and heat transfer in a narrow TaylorCouette-Poiseuille system". Int. J. Heat Fluid Flow, 32, pp. 128-144.

[7] Rothe, T., and Pfitzer, H., 1997. "The influence of rotation on turbulent flow and heat transfer in an annulus between independently rotating tubes". Int. J. Heat Mass Transfer, 32, pp. 353-364.

[8] Chung, S., and Sung, H., 2005. "Large-eddy simulation of turbulent flow in a concentric annulus with rotation of an inner cylinder". Int. J. Heat Fluid Flow, 26, pp. 191-203.

[9] Hadziabdic, M., Hanjalić, K., and Mullyadzhanov, R., 2013. "LES of turbulent flow in a concentric annulus with rotating outer wall". Int. J. Heat Fluid Flow, 43, pp. 74-84.

[10] Oguic, R., Viazzo, S., and Poncet, S., 2013. "Numerical simulations of a middle gap turbulent Taylor-CouettePoiseuille flow". In Direct and Large-Eddy Simulation 9, Dresden.

[11] Friess, C., Poncet, S., and Viazzo, S., 2013. "TaylorCouette-Poiseuille flows : from RANS to LES". Proc. 8th Int. Symp. Turb. Shear Flow Phenomena, Poitiers.

[12] Abide, S., and Viazzo, S., 2005. "A 2D compact fourthorder projection decomposition method". J. Comput. Phys., 206, pp. 252-276.

[13] Viazzo, S., Poncet, S., Serre, E., Randriamampianina, A., and Bontoux, P., 2012. "High-order Large Eddy Simulations of confined rotor-stator flows". Flow, Turb. \& Comb., $88(1-2)$, pp. 63-75.

[14] Archambeau, F., Méchitoua, N., and Sakiz, M., 2004. "Code Saturne: A finite volume code for the computation of turbulent incompressible flows - Industrial applications". Int. J. Finite Volume, Electronical edition: http://averoes.math.univ-paris13.fr/html, ISSSN
1634(0655).

[15] Manceau, R., Friess, C., and Gatski, T., 2010. "Of the interpretation of DES as a hybrid RANS / temporal LES method". In 8th ERCOFTAC Int. Symp. Eng. Turbulence Modelling Measurements, Marseille.

[16] Chaouat, B., and Schiestel, R., 2005. "A new partially integrated transport model for subgrid-scale stresses and dissipation rate for turbulent developing flows". Phys. Fluids, 17(065106), pp. 1-19.

[17] Fadai-Ghotbi, A., Friess, C., Manceau, R., Gatski, T., and Borée, J., 2010. "Temporal filtering : A consistent formalism for seamless hybrid RANS/LES in inhomogeneous turbulence". Int. J. Heat Fluid Flow, 31, pp. 378-389.

[18] Manceau, R., 2005. "An improved version of the Elliptic Blending Model. Application to non-rotating and rotating channel flows". In Proc. 4th Int. Symp. Turb. Shear Flow Phenomena, Williamsburg.

[19] Fadai-Ghotbi, A., Friess, C., Manceau, R., and Borée, J., 2010. "A seamless hybrid RANS/LES model based on transport equations for the subgrid stresses and elliptic blending". Phys. Fluids, 22, p. 055104.

[20] Dehoux, F., Benhamadouche, S., and Manceau, R., 2011. "Modelling turbulent heat fluxes using the elliptic blending approach for natural convection". In Proc. 7th Int. Symp. Turb. Shear Flow Phenomena, Ottawa.

[21] Nicoud, F., and Ducros, F., 1999. "Subgrid-scale stress modelling based on the square of the velocity gradient tensor". Flow, Turb. \& Comb., 62, pp. 183-200.

[22] Germano, M., Piomelli, U., Moin, P., and Cabot, W., 1991. "A dynamic subgrid-scale eddy-viscosity model". Phys. Fluids A: Fluid Dynamics, 3(7), pp. 1760-1765.

[23] Lilly, D., 1992. "A Proposed Modification of the Germano Subgrid Scale Closure Method”. Phys. Fluids A: Fluid Dynamics, 4(3), pp. 633-635.

[24] Poncet, S., 2014. Instabilities, turbulence and heat transfer in confined rotating flows - habilitation thesis. Tech. rep., Aix-Marseille University. 
Appendix A: Wall resolution and mean flow parameters

\begin{tabular}{|c|c|c|c|c|c|c|c|c|c|c|c|c|}
\hline \multirow{3}{*}{$\begin{array}{c}\text { Case } \\
N\end{array}$} & \multirow{2}{*}{\multicolumn{4}{|c|}{$\begin{array}{c}\mathrm{D} \\
1.49\end{array}$}} & \multirow{2}{*}{\multicolumn{4}{|c|}{$\begin{array}{c}\mathrm{A} \\
2.24\end{array}$}} & \multicolumn{4}{|c|}{$\mathrm{E}$} \\
\hline & & & & & & & & & \multicolumn{4}{|c|}{2.98} \\
\hline & LES-W & LES-DS & HRLES & RANS & LES-W & LES-DS & HRLES & RANS & LES-W & LES-DS & HRLES & RANS \\
\hline$r_{i}^{+}$ & 0.38 & 0.38 & 0.74 & 0.63 & 0.47 & 0.48 & 0.63 & 0.51 & 0.52 & 0.52 & 1.10 & 0.86 \\
\hline$r_{o}^{+}$ & 0.34 & 0.34 & 0.67 & 0.63 & 0.43 & 0.44 & 0.57 & 0.51 & 0.47 & 0.47 & 1.00 & 0.84 \\
\hline$\left(R_{1} \Delta \theta\right)^{+}$ & 57.66 & 57.64 & 102.3 & - & 44.45 & 44.70 & 86.73 & - & 78.62 & 79.31 & 151.6 & - \\
\hline$\left(R_{2} \Delta \theta\right)^{+}$ & 58.01 & 59.05 & 103.3 & & 45.27 & 45.90 & 87.64 & - & 80.48 & 80.65 & 155.0 & - \\
\hline$\Delta z_{i}^{+}$ & 22.00 & 21.99 & 81.42 & & 26.95 & 27.10 & 69.02 & - & 29.99 & 30.26 & 120.7 & - \\
\hline$\Delta z_{o}^{+}$ & 19.68 & 20.02 & 73.05 & - & 24.40 & 24.74 & 61.99 & & 27.29 & 27.34 & 109.7 & - \\
\hline $\operatorname{Re}_{\tau, i}$ & 391.6 & 391.4 & 488.5 & 411.9 & 339.6 & 341.6 & 414.1 & 333.4 & 533.8 & 538.6 & 724.1 & 567.3 \\
\hline $\operatorname{Re} \tau, o$ & 350.2 & 356.4 & 438.3 & 412.0 & 307.4 & 311.6 & 372.0 & 333.3 & 485.8 & 486.8 & 658.0 & 551.8 \\
\hline$N_{r}$ & 91 & 91 & 60 & 60 & 65 & 65 & 60 & 60 & 91 & 91 & 60 & 60 \\
\hline$N_{\theta}$ & 128 & 128 & 60 & 1 & 144 & 144 & 60 & 1 & 128 & 128 & 60 & 1 \\
\hline$N_{z}$ & 182 & 182 & 60 & 1 & 130 & 130 & 60 & 1 & 182 & 182 & 60 & 1 \\
\hline$\delta_{t} W_{m} /\left(R_{2}-R_{1}\right)$ & \multicolumn{2}{|c|}{$3.5 \times 10^{-4}$} & $4.2 \times 10^{-2}$ & - & \multicolumn{2}{|c|}{$1.2 \times 10^{-3}$} & \multicolumn{2}{|l|}{$2.8 \times 10^{-2}$} & \multicolumn{2}{|c|}{$8.3 \times 10^{-4}$} & $2.1 \times 10^{-2}$ & - \\
\hline Azimuthal periodicity (deg) & 135 & 135 & 90 & 90 & 135 & 135 & 90 & 1.5 & 135 & 135 & 90 & 1.5 \\
\hline Case & \multirow{2}{*}{\multicolumn{4}{|c|}{$\begin{array}{c}\text { B } \\
4.47\end{array}$}} & \multirow{2}{*}{\multicolumn{4}{|c|}{$\begin{array}{c}F \\
4.47\end{array}$}} & \multirow{2}{*}{\multicolumn{4}{|c|}{$\begin{array}{c}\text { C } \\
6.71\end{array}$}} \\
\hline$N$ & & & & & & & & & & & & \\
\hline method & LES-W & LES-DS & HRLES & RANS & LES-W & LES-DS & HRLES & RANS & LES-W & LES-DS & HRLES & RANS \\
\hline$r_{i}^{+}$ & 0.66 & 0.67 & 1.02 & 0.76 & 0.62 & 0.65 & 1.46 & 1.09 & 0.86 & 0.86 & 1.45 & 1.04 \\
\hline$r_{o}^{+}$ & 0.60 & 0.60 & 0.92 & 0.74 & 0.62 & 0.65 & 1.23 & 1.09 & 0.76 & 0.77 & 1.29 & 1.04 \\
\hline$\left(R_{1} \Delta \theta\right)^{+}$ & 62.00 & 62.76 & 140.8 & & 94.76 & 98.53 & 200.6 & - & 80.51 & 80.54 & 199.3 & - \\
\hline$\left(R_{2} \Delta \theta\right)^{+}$ & 62.86 & 63.33 & 142.1 & - & 96.05 & 99.28 & 191.3 & - & 80.49 & 81.22 & 199.7 & - \\
\hline$\Delta z_{i}^{+}$ & 37.60 & 38.05 & 112.1 & - & 36.15 & 37.59 & 159.6 & & 48.82 & 48.83 & 158.6 & - \\
\hline$\Delta z_{o}^{+}$ & 33.88 & 34.13 & 100.5 & & 32.57 & 33.67 & 135.3 & - & 43.38 & 43.77 & 141.2 & - \\
\hline$R e_{\tau, i}$ & 473.8 & 479.4 & 672.3 & 501.7 & 643.4 & 668.6 & 957.9 & 717.8 & 615.0 & 615.4 & 951.7 & 683.2 \\
\hline $\operatorname{Re} \tau, o$ & 426.8 & 430.0 & 603.1 & 486.3 & 579.8 & 599.2 & 811.8 & 717.8 & 546.6 & 551.6 & 847.4 & 683.1 \\
\hline$N_{r}$ & 65 & 65 & 60 & 60 & 91 & 91 & 60 & 60 & 65 & 65 & 60 & 60 \\
\hline$N_{\theta}$ & 144 & 144 & 60 & 1 & 128 & 128 & 60 & 1 & 144 & 144 & 60 & 1 \\
\hline$N_{z}$ & 130 & 130 & 60 & 1 & 182 & 182 & 60 & 1 & 130 & 130 & 60 & 1 \\
\hline$\delta t W_{m} /\left(R_{2}-R_{1}\right)$ & \multicolumn{2}{|c|}{$7.9 \times 10^{-4}$} & $1.4 \times 10^{-2}$ & & \multicolumn{2}{|c|}{$5.9 \times 10^{-4}$} & $1.4 \times 10^{-2}$ & . & \multicolumn{2}{|c|}{$6.3 \times 10^{-4}$} & $9.3 \times 10^{-3}$ & \\
\hline Azimuthal periodicity (deg) & 135 & 135 & 90 & 1.5 & 135 & 135 & 90 & 1.5 & 135 & 135 & 90 & 1.5 \\
\hline
\end{tabular}

TABLE 2. Computational details and mean flow parameters. 